\title{
Electromagnetic Analysis and Validation of an Ironless Inductive Position Sensor
}

\author{
Alessandro Danisi, Graduate Student Member, IEEE, Alessandro Masi, Roberto Losito, and \\ Yves Perriard, Senior Member, IEEE
}

\begin{abstract}
The ironless inductive position sensor is a linear position sensing structure, which exhibits intrinsic immunity to external magnetic fields since it is characterized by air-cored windings. This new solution may be of major interest for applications where external magnetic fields can be a source of interference. In this paper, an analytical model of the working principle of the sensor is proposed. The effect of the moving coil flux on the overall sensed magnetic flux is described. The model is preliminarily verified by simulations on a finite-element structure of the sensor, in order to assess its soundness. Finally, experimental measurements on a custom sensor's prototype give the definitive benchmarking of the model as a valid design tool, in the framework of the design and synthesis of the device.
\end{abstract}

Index Terms-Air-cored coils, electromagnetic modeling, ironless position sensor, linear variable differential transformer (LVDT), mutual inductance.

\section{INTRODUCTION}

$\mathbf{L}$ INEAR position sensors are often adopted for measurements in harsh environments, as in particle accelerators and nuclear plants [1], [2], where precisions up to some micrometers over centimeter ranges are often required [3]. For this purpose, linear variable differential transformers (LVDTs) are often the preferred choice. As a matter of fact, these inductive position sensors exhibit excellent precision performances with proper reading algorithm [3], virtually infinite resolution, long lifetime, and good robustness [2], [4]; and can be set up to be radiation hard with proper insulation and sealing techniques [1], [5]. The relatively low cost and wide range (from a few millimeters up to a meter) make them a frequent choice also for common applications such as automotive and industry [4]. Nevertheless, LVDT sensors show significant measurement drifts when an external magnetic field is applied in the longitudinal direction (i.e., parallel to the sensor's axis) [6], [7]. Position drifts of some hundreds of micrometers have been observed

Manuscript received June 20, 2012; revised September 21, 2012; accepted September 22, 2012. Date of publication October 26, 2012; date of current version April 3, 2013. The Associate Editor coordinating the review process for this paper was Dr. Subhas Mukhopadhyay.

A. Danisi is with the Engineering Department/Sources, Targets and Interaction Group, European Organization for Nuclear Research (CERN), 1211 Geneva, Switzerland, and also with the Institute of MicroengineeringLaboratoire d'Actionneurs Intégrés, Ecole Polytechnique Fédérale de Lausanne, 2000 Neuchâtel, Switzerland (e-mail: Alessandro.Danisi@cern.ch).

A. Masi and R. Losito are with the Engineering Department/Sources, Targets and Interaction Group, European Organization for Nuclear Research (CERN), 1211 Geneva, Switzerland.

Y. Perriard is with the Institute of Microengineering-Laboratoire d'Actionneurs Intégrés, Ecole Polytechnique Fédérale de Lausanne, 2000 Neuchâtel, Switzerland.

Digital Object Identifier 10.1109/TIM.2012.2224274 with dc/slowly varying longitudinal magnetic fields [7]. These drifts are often an unacceptable drawback in high-precision applications [8]. On the other hand, a transversal magnetic field has shown negligible influence on the LVDT position reading [6].

Analytical study of the phenomenon [9], characterizations through finite-element method (FEM) simulations [7], [10], and experimental measurement campaigns [7], [11] have demonstrated the unavoidability of this effect for typical LVDT structures due to the presence of ferromagnetic materials with a nonlinear B-H relation, which are part of the intrinsic design of an LVDT. Such nonlinearity between the field and flux densities makes even a dc or slowly varying magnetic field affect the harmonics of the sensor windings' voltages, therefore affecting the position reading.

The ironless inductive position sensor (I2PS) has been proposed in [12] to overcome the LVDT magnetic interference problem by designing a sensing structure that is intrinsically immune to external magnetic fields. This task is achieved by avoiding the use of magnetic materials. In addition, with the working principle being based anyway on inductive coupling between non-contact windings, the sensor keeps the main advantages of typical LVDTs [12], and it qualifies as a valid alternative for magnetic environments.

The effectiveness of the I2PS as a position sensor and its immunity to external dc and slowly varying magnetic fields have been already shown [12]. In addition, the analytical model that describes the sensor's working principle and demonstrates the dependence of the flux linkage with the position, from a theoretical point of view, has been presented in [13], together with a first verification through FEM simulations. This paper aims at the full validation of the electromagnetic model, with more detailed simulations and particularly through experimental measurements, on an I2PS prototype, which has been explicitly manufactured for this purpose. In addition, significant improvements are proposed for the computational time efficiency of the model. Once the complete validation is performed, the resulting model would be a powerful tool, from the electromagnetic point of view, for the sensor's analysis, study, optimization, and design.

In Section II, the ironless position sensor is briefly described, and a comparison with the working principle of the LVDT is explained. In Section III, the model is discussed in detail for voltage and current supply cases. In Section IV, the finiteelement model of the sensor and the preliminary validation are presented. In Section V, the measurement setups and results and their comparison with the proposed model predictions are discussed. 

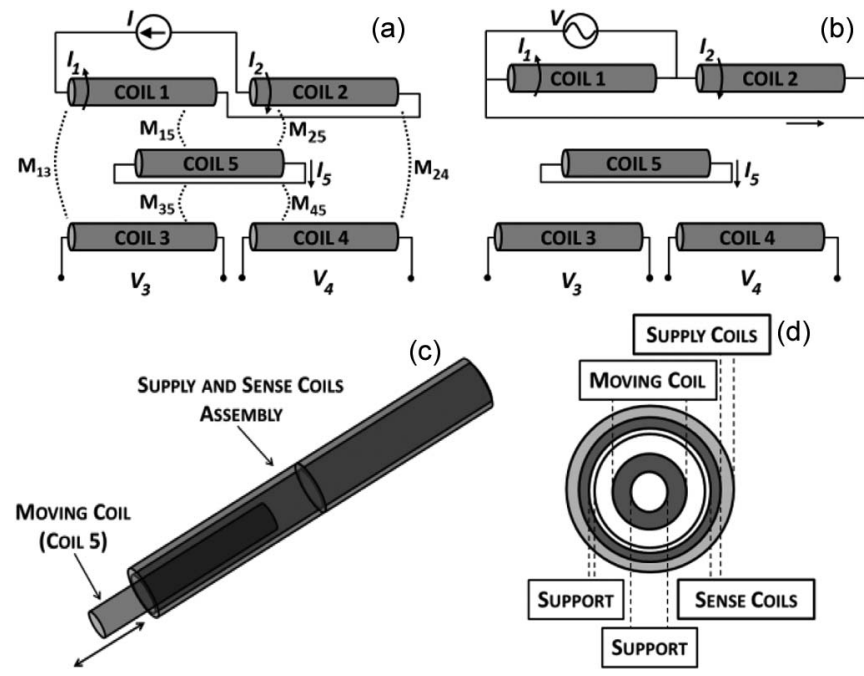

Fig. 1. (a) I2PS structure and its working principle in current supply. The dotted lines connecting the coils indicate the mutual coupling. Coils 1 and 2 are the supply coils, coils 3 and 4 are the sense coils, and coil 5 is the moving coil. (b) Structure with voltage supply (the mutual inductances are omitted). (c) Sensor's assembly in three dimensions. All coils are coaxial. (d) Transversal view showing the arrangement of the coils and the winding supports.

\section{IRONLESS POSITION SENSOR}

The structure of an I2PS is depicted in Fig. 1. It is a fivecoil coaxial assembly with two supply coils (coils 1 and 2), two sense coils (coils 3 and 4), and a moving coil (coil 5). The two supply coils' voltages are set to be in opposition of phase so that the corresponding magnetic fluxes are equal and opposite. The net current $I_{5}$ induced in the moving coil (which is short circuited) depends on the coil position itself and so does the resulting counter flux produced by this current. This acts as a perturbation on the flux linkage between the supply and sense coils, depending on the moving coil position. By reading the difference of the two sense coils' voltages (just as for LVDTs [2], [4]) the position of the moving coil can be extracted.

As the LVDT, the I2PS works by spatially varying the magnetic flux coupled with the windings, with respect to different positions of a moving object [12]. The difference is that the moving coil of the I2PS perturbs the flux linkage between the supply and sense coils, whereas the moving core of the LVDT increases it [4]. In particular, in the LVDT, the ferromagnetic core increases the mutual inductance between the primary and secondary windings; whereas the moving coil of the I2PS generates its own magnetic field (due to the induced current in the coil), which counteracts the one due to the supply signal. The strength of this counteraction depends on the induced current, which, in turn, depends on the position. Therefore, the magnetic flux is modulated according to the position and so are the induced voltages on the windings. The geometry of the I2PS keeps the main advantages of LVDT sensors since there is contactless sensing (which leads to a long lifetime), virtually infinite resolution, good precision (the reading can be performed as for LVDTs), robustness, and possibility to implement radiation hardness. Some of these features are intrinsically present in the sensor; some can be achieved with proper optimization, insulation, and design.

\section{Electromagnetic Model}

The calculation of the mutual and self-inductances of the different windings is the starting point of the full analytical description of the I2PS working principle. The model takes into account the following hypotheses as references: 1) Time transient effects are not considered; 2) the signals are considered purely sinusoidal, although the model equations can be applied even to each harmonic of a multitone signal; and 3) highfrequency phenomena, such as skin and proximity effects and the presence of parasitic winding capacitances, are neglected. For the first two hypotheses, it is possible to write the equations directly in the phasors' domain.

\section{A. Mutual and Self-Inductances Computation}

Starting from the parameters of the windings, such as number of turns, number of layers, length, and winding section, the values of mutual and self-inductances can be accurately calculated. As evident in Fig. 1(d), all windings are coaxial. Given the absence of magnetic materials, the mutual inductances will only depend on the windings' geometry and their relative position along the axis (i.e., position of the moving coil, being this the only movable part, as shown in Fig. 1). The mutual inductance between the sensor's thick windings (i.e., windings with several layers) has been calculated, adding up the mutual inductances between the single layers. The mutual inductance between two single layers has been, in turn, calculated, adding up the contribution of elementary mutual inductances between two circular coaxial coils (filament method [14]-[16]). Such elementary contributions are given by [15]

$$
\begin{aligned}
M_{i} & =\frac{2 \mu_{0} \sqrt{R_{p} R_{s}}}{k}\left[\left(1-\frac{k^{2}}{2}\right) K(k)-E(k)\right] \\
& =\mu_{0} \sqrt{R_{p} R_{s}} \phi(k)
\end{aligned}
$$

where $\mu_{0}=4 \pi \cdot 10^{-7} \mathrm{H} / \mathrm{m}, R_{s}$ and $R_{p}$ are the coil radii, and

$$
\begin{aligned}
\alpha & =\frac{r_{s}}{R_{p}}, \quad \beta=\frac{c}{R_{p}}, \quad k^{2}=\frac{4 \alpha}{(1+\alpha)^{2}+\beta^{2}} \\
\phi(k) & =\left(\frac{2}{k}-k\right) K(k)-\frac{2}{k} E(k)
\end{aligned}
$$

with $c$ being the distance between the elementary coils (see Fig. 2). $K$ and $E$ are the elliptic integrals of first and second kind, respectively. Therefore, letting $N_{l p}$ and $N_{l s}$ be the number of layers of the first and second windings and $N_{p}$ and $N_{s}$ be the number of turns per layer of the first and second windings, respectively, the overall mutual inductance for the two coils at distance $c$ is

$$
M_{\tau}(c)=\mu_{0} \sqrt{R_{p} R_{s}} \sum_{i=1}^{N_{l p}} \sum_{j=1}^{N_{l s}} \sum_{n=1}^{N_{p}} \sum_{l=1}^{N_{s}} \phi i, j, n, l\left(k_{i, j, n, l}\right)
$$

where $\phi_{i, j, n, l}$ and $k_{i, j, n, l}$ have been defined in (2) and assume different values according to the turn or the layer.

In the case of a mutual inductance involving the moving coil, the distance $c_{0}$ between the thick-walled coils (see Fig. 2) will 


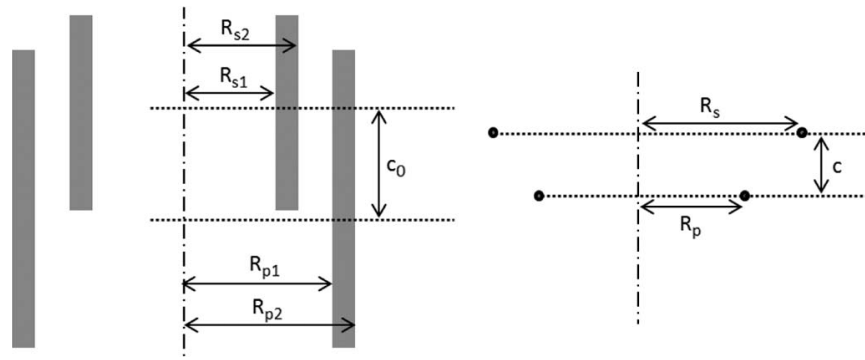

Fig. 2. (Left) Two cylindrical thick-walled solenoids with different centers. (Right) Two elementary coaxial circular coils with different centers.

be proportional to the moving coil position, and the resulting mutual inductance will depend on that. The computations carried out in [13] have shown that this dependence is linear.

The self-inductance is seen as the superposition of the selfinductances of each layer and the mutual inductances between the layers. The self-inductance of a layer is computed as [17]

$$
L_{l}=\frac{\mu_{0} \pi N^{2} R^{2}}{2 s} T\left(k_{l}\right)
$$

where $N$ is the number of turns, $R$ the layer radius, $s$ is the winding semi-length, and

$$
\begin{aligned}
T\left(k_{l}\right) & =\frac{4}{3 \pi \beta k_{l}^{3}}\left[\left(2 k_{l}^{2}-1\right) E\left(k_{l}\right)+\left(1-k_{l}^{2}\right) K\left(k_{l}\right)-k_{l}^{3}\right] \\
k_{l}^{2} & =\left(1+\beta^{2}\right)^{-1}
\end{aligned}
$$

where $\beta$ is a shape factor [17]. The mutual inductance between the layers (i.e., the second term to consider to calculate the selfinductance) is computed using (1).

The mutual inductance expressed in (3) involves the computation of an elementary mutual inductance, which is expressed in (1), for $G=N_{l p} \times N_{l s} \times N_{p} \times N_{s}$ times. The computation of such elementary contribution entails, in turn, the evaluation of elliptic integrals (which can be numerically carried out). For the sensor's design, $G$ can be a very big number (e.g., on the order of $10^{7}$ ), which leads to a possibility to have a very high computational time for the mutual inductances. Finally, given the presence of five windings, the computation of all the mutual inductances at all the desired position values can be a seriously time-consuming task. The criticality of such computational time also lies in the fact that the model has to be a valid tool for the sensor's design, which includes repeated evaluations for optimization, fast counterchecks, and rapid parametric analysis.

For this reason, an approximated computation of the total mutual inductances is possible by defining an iteration scaling factor, i.e.,

$$
s=\frac{G^{\mathrm{app}}}{G^{\mathrm{comp}}}
$$

where $G^{\text {app }}$ and $G^{\text {comp }}$ are the number of iterations (i.e., the number of evaluations of elementary mutual inductance) in the approximated and precise calculations, respectively. The computation of the mutual inductance can be then performed, applying the scaling factor, to have

$$
G^{\text {app }}=N_{l p} \times N_{l s} \times \sqrt{s} N_{p} \times \sqrt{s} N_{s} .
$$

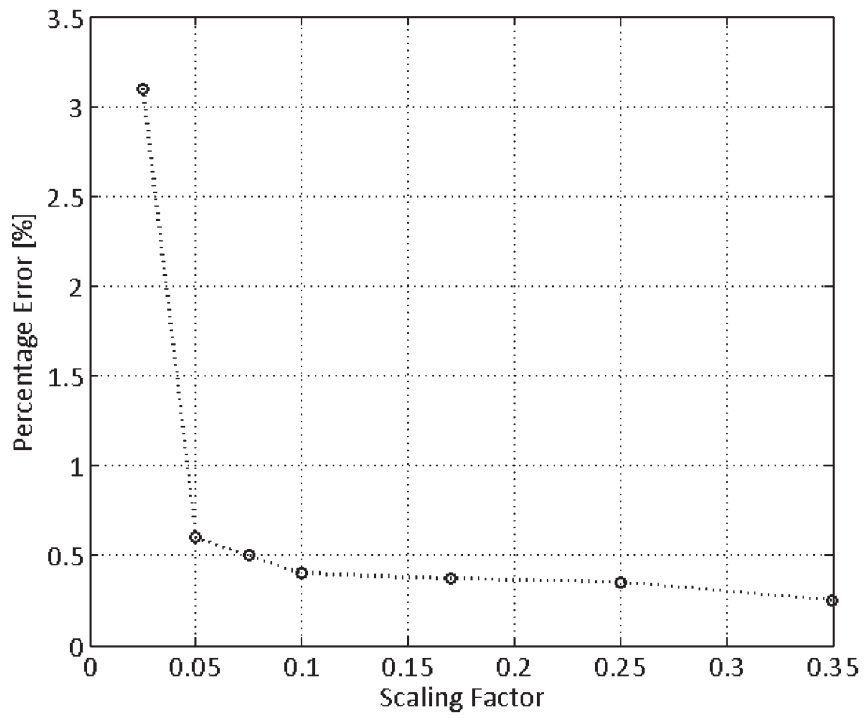

Fig. 3. Computation error versus scaling factor for the approximated calculation of the total mutual inductance.

The resulting mutual inductance can be then divided by $s$ to have the approximate value. This operation is equivalent to physically reduce the number of turns per layer and assume that the mutual inductance is approximately linear with $N_{p} N_{s}$ for big values of this product. The scaling factor has been applied to the number of turns per layer, being this the biggest number in the factors forming $G$. By doing so, the computational time is substantially reduced.

Fig. 3 shows the approximation error, which is computed as the percentage difference between the values of mutual inductance obtained with and without the application of the scaling factor, for different values of the latter. For low values, the product $N_{p} N_{s}$ and the winding arrangement are substantially modified. The error attains 3\% in this case, whereas for more reasonable values, it always stays under $0.5 \%$. Therefore, to assure a good computational time and, at the same time, not to make a relevant error, the scaling factor should be the result of a compromise. As an example, choosing a scaling factor of 0.1 has led to a computational time saving of more than $80 \%$.

Therefore, using (3) and (4), all mutual and self-inductances of the five-winding structure in Fig. 1 can be calculated for different values of the moving coil position. The electrical quantities have to be found at the next stage.

\section{B. Derivation of Electrical Magnitudes}

Henceforth, the number labels shown in Fig. 1 are adopted to refer to the single windings. The moving coil being short circuited, by simply applying the voltage balance on its mesh and considering all the magnetic fluxes coupled with the coil, one can obtain the induced current, i.e.,

$$
I_{5}=\frac{j \omega}{Z_{5}}\left(M_{51} I_{1}+M_{52} I_{2}\right)
$$

where $Z_{i}=R_{i}+j \omega L_{i}$ is the winding impedance (in the case of the moving coil, the impedance of the short-circuited winding), $M_{i j}$ is the mutual inductance between windings $i$ and $j$, 
and $I_{i}$ is the current in coil $i$. Equation (7) assumes that no current is flowing in the sense windings. In fact, they should be connected to the high-impedance readout system and, therefore, characterized by a very small (less than a microampere) current.

The sense voltages are a function of the magnetic flux generated by the supply coils and the flux due to the current flowing in the short-circuited moving coil. As a result, the sense voltages are a function of the supply currents and the current in the moving coil, which are as follows:

$$
\begin{aligned}
& V_{3}=j \omega\left(M_{31} I_{1}+M_{32} I_{2}+M_{35} I_{5}\right) \\
& V_{4}=j \omega\left(M_{42} I_{2}+M_{41} I_{1}+M_{45} I_{5}\right) .
\end{aligned}
$$

There is no dependence on the self-inductances of the sense coils since no current is flowing through them, as mentioned. Substituting (7) into (8), the sense voltages can be expressed as

$$
\begin{array}{r}
V_{3}=j \omega\left(M_{31} I_{1}+M_{32} I_{2}-\frac{j \omega M_{35}}{Z_{5}}\right. \\
\left.+M_{51} I_{1}-\frac{j \omega M_{35}}{Z_{5}} M_{52} I_{2}\right) \\
V_{4}=j \omega\left(M_{42} I_{2}+M_{41} I_{1}-\frac{j \omega M_{45}}{Z_{5}}\right. \\
\left.+M_{51} I_{1}-\frac{j \omega M_{45}}{Z_{5}} M_{52} I_{2}\right) .
\end{array}
$$

In (9), all mutual inductances that involve winding number 5 depend on the position since they refer to the moving coil. These relations are general since they are valid in any supply condition (voltage or current).

In the simple case of current supply [see Fig. 1(a)], $I_{2}=$ $-I_{1}=I$. The supply voltages, considering all the magnetic fluxes, can be written as

$$
\begin{aligned}
& V_{1}=R_{1} I+j \omega\left(L_{1}-M_{12}\right) I+j \omega M_{15} I_{5} \\
& V_{2}=-R_{2} I+j \omega\left(M_{21}-L_{2}\right) I+j \omega M_{25} I_{5}
\end{aligned}
$$

whereas the sense voltages can be obtained, starting from (9) and considering $I_{2}=-I_{1}=I$, as follows:

$$
\begin{aligned}
& V_{3}=j \omega\left(M_{31}-M_{32}\right) I+\omega^{2} I\left(\frac{M_{35} M_{51}}{Z_{5}}-\frac{M_{35} M_{52}}{Z_{5}}\right) \\
& V_{4}=j \omega\left(M_{42}-M_{41}\right) I+\omega^{2} I\left(\frac{M_{45} M_{51}}{Z_{5}}-\frac{M_{45} M_{52}}{Z_{5}}\right) .
\end{aligned}
$$

Equation (11) show that the sense voltages can be seen as the superposition of the induced voltage due to the primary current (first term), which is independent from the position, and the perturbing voltage due to the moving coil counter flux (second term), which gives the dependence on the position. As a matter of fact, if the moving coil was absent, the second term in (11) would be null. This term would also vanish if the moving coil was open circuited. As a matter of fact, in this particular case, there would not be an induced current in such coil, and the expression for the sense voltage could be derived, imposing $\left|Z_{5}\right| \rightarrow \infty$. The short-circuiting of the moving coil is then a necessary condition for the sensor to work properly.
As one can notice, (10) prove that the voltages on the supply windings depend, in turn, on the moving coil position. In fact, they can be seen as the superposition of a constant term (due to the resistance and mutual and self-inductances between the supply coils) and a second term (due to the perturbation of the moving coil flux). For the sake of completeness, a more complete expression of the supply voltages can be obtained by substituting (7) into (10), i.e.,

$$
\begin{aligned}
& V_{1}=R_{1} I+j \omega\left(L_{1}-M_{12}\right) I+\frac{\omega^{2} I}{Z_{5}} M_{15}\left(M_{51}-M_{52}\right) \\
& V_{2}=-R_{2} I+j \omega\left(M_{21}-L_{2}\right) I+\frac{\omega^{2} I}{Z_{5}} M_{25}\left(M_{51}-M_{52}\right) .
\end{aligned}
$$

Equations (11) and (12) describe the voltage on all windings for current supply.

In case of voltage supply [see Fig. 1(b)], $V_{2}=-V_{1}=V$. In this case, the formulation becomes more complicated since the supply currents are now depending on the position. In particular, considering all the coupled magnetic fluxes, the supply currents can be written as

$$
\begin{aligned}
& I_{1}=\frac{V}{Z_{1}}-j \omega \frac{M_{12} I_{2}}{Z_{1}}-j \omega \frac{M_{15} I_{5}}{Z_{1}} \\
& I_{2}=\frac{-V}{Z_{2}}-j \omega \frac{M_{21} I_{1}}{Z_{2}}-j \omega \frac{M_{25} I_{5}}{Z_{2}}
\end{aligned}
$$

where the first terms are due to the winding impedance, the second terms are due to the parasitic coupling between the supply windings, and the third terms are given by the coupling with the moving coil. This last term gives the dependence on the position. Equations (13) and (7) constitute a system of three linearly independent equations with three unknowns (the three currents $I_{1}, I_{2}$, and $I_{5}$ ). Once solved, the moving coil current can be expressed as

$$
I_{5}=\frac{\left(\frac{V \cdot M_{52}}{Z_{2}} A_{2}-\frac{V \cdot M_{51}}{Z_{1}} A_{1}\right) \cdot C}{1-\omega C\left(\frac{M_{51}}{Z_{1}} B_{1}+\frac{M_{52}}{Z_{2}} B_{2}\right)}
$$

where

$$
\begin{aligned}
& A_{1}=1+j \omega \frac{M_{12}}{Z_{2}}, \quad A_{2}=1+j \omega \frac{M_{21}}{Z_{1}} \\
& B_{1}=j M_{15}+\omega \frac{M_{12} M_{25}}{Z_{2}}, \quad B_{2}=j M_{25}+\omega \frac{M_{21} M_{15}}{Z_{1}} \\
& C=\frac{j \omega}{Z_{5}\left(1+\omega^{2} \frac{M_{12}^{2}}{Z_{1} Z_{2}}\right)}
\end{aligned}
$$

whereas the two supply currents are

$$
\begin{aligned}
& I_{1}=\frac{\frac{V}{Z_{1}} A_{1}-\frac{V}{Z_{1}} \omega \cdot C \frac{M_{52}}{Z_{2}}\left(A_{1} B_{2}+A_{2} B_{1}\right)}{\frac{j \omega}{Z_{5} C}\left[1-\omega \cdot C\left(\frac{M_{51}}{Z_{1}} B_{1}+\frac{M_{52}}{Z_{2}} B_{2}\right)\right]} \\
& I_{2}=\frac{\frac{-V}{Z_{2}} A_{2}+\frac{V}{Z_{2}} \omega \cdot C \frac{M_{51}}{Z_{1}}\left(A_{1} B_{2}+A_{2} B_{1}\right)}{\frac{j \omega}{Z_{5} C}\left[1-\omega \cdot C\left(\frac{M_{51}}{Z_{1}} B_{1}+\frac{M_{52}}{Z_{2}} B_{2}\right)\right]} .
\end{aligned}
$$


Simple considerations can be done concerning (15) and (14) in order to verify their consistence. If all mutual inductances were null (i.e., no magnetic coupling between windings), the terms $A_{i}$ and $B_{i}$ would approach zero, the term $C$ would go to $j \omega / Z_{5}$, and then the currents would be

$$
I_{1}=\frac{V}{Z_{1}}, \quad I_{2}=\frac{-V}{Z_{2}}, \quad I_{5}=0
$$

which is consistent with the hypothesis. In practice, this situation would describe the currents of three independent electrical circuits. Once again, if the moving coil was open circuited, $I_{5}$ would vanish in (13), and there would be no dependence on the position.

The two sense coils' voltages can be finally found by substituting (14) and (15) into the general expression (8). To make a comparison with the current supply case, it is evident that, in voltage supply, the effect of the moving coil on the sense coils acts in two manners: It perturbs the flux linkage between the supply and sense coils (as for current supply), but it also modulates the supply currents, which, in turn, affects the sense voltages. On the other hand, the analysis in current supply shows that the effect of the moving coil acts both on the sense and supply coils' voltages. Nevertheless, there is no direct relation between the supply and sense voltages in current supply; thus, the two effects are separated.

The immunity to external $\mathrm{dc}$ magnetic fields is intrinsic to the design of the sensor's working principle and coil assembly since such a field would not yield any voltage contribution. From the point of view of the model, since the analysis has been performed in the phasors' domain, a dc field cannot have influence on the analyzed magnitudes. On the other hand, an ultralow-frequency interfering sinusoidal field would actually give rise to sinusoidal voltages on the windings, according to Faraday's law. Nevertheless, such components would also have no effect on the sensor's reading since this is performed by selecting the specific harmonics of the windings' voltages at the excitation frequency (i.e., some kilohertzes). Therefore, the low-frequency components will be filtered out, and no cross effect on the main harmonics will be present, with the whole system described in the model being linear. These performances have been experimentally observed in [12] and cannot be obtained with LVDTs due to the nonlinear ferromagnetic materials, whose permeability is modulated by the external field [7], [9], [10].

\section{FEM PRELIMINARY VALIDATION}

A finite-element model of the ironless position sensor has been built using the FEM simulator Flux in the 2-D environment: Given the cylindrical symmetry of the geometry in terms of magnetic and electrical magnitudes, this environment is sufficient for performing the analysis.

The FEM simulations are the ideal mean to perform a first validation of the model since, in the simulations, there is no influence from other physical magnitudes, such as temperature. Of course, the simulator takes into account the parasitic effects due to capacitances; therefore, the assumption made in the model treatise to neglect them can be also verified. Finally, the

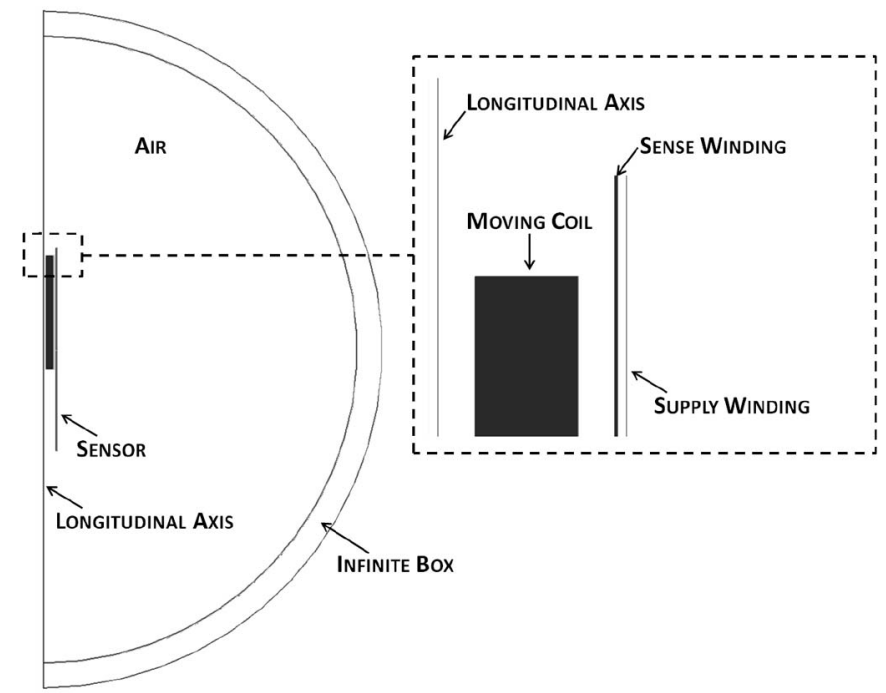

Fig. 4. Simulation geometry with the moving coil in a nonnull position. Each rectangular region is the longitudinal cross section of a winding. In the square is a detail of the coil's arrangement. Given the cylindrical symmetry, half of the longitudinal section is depicted. The 3-D structure is obtained by rotating the 2-D structure around the longitudinal axis.

FEM analysis allows the investigation on physical magnitudes, which are otherwise difficult to measure, such as the induced current in the moving coil. For these reasons, the validation through FEM simulations will mainly concern the currents in the windings (particularly the moving coil) and the supply voltages amplitudes, which is basically to validate the soundness and reliability of the model. The complete comparison with regard to the sense voltages will be addressed in detail in Section V, where the definitive validation of the sensor's working principle is proposed.

For the geometry and dimensions, no optimization has been done since the only aim of the simulations is to validate the analytical model. The optimization is a later stage. However, the simulations and the model equations have been compared on a wider moving coil position range with respect to [13], in order to perform the comparison also near the edges.

\section{A. FEM Model}

The axisymmetric structure prepared for the simulations is depicted in Fig. 4. If rotated around the longitudinal axis, it gives the 3-D structure already depicted in Fig. 1(c). The axis represents a boundary, in which a condition of symmetry has to be imposed (tangential magnetic field). The open space is reconstructed with an infinite box, where a geometrical transformation is performed to simulate the infinite space and assign the null-field boundary conditions [18].

Given the high ratio between lengths and diameters, a fine mesh has been set up. The number of surface elements is 20000 . In principle, the number of mesh elements can be reasonably high since, in the solving process, the nonlinear solver will be bypassed (all B-H curves are linear), and thus, the computational time is not a significant concern.

The windings' sections have been modeled with rectangular windows, without taking into account the single wire cross sections. However, a fill factor can be specified [18]. The 

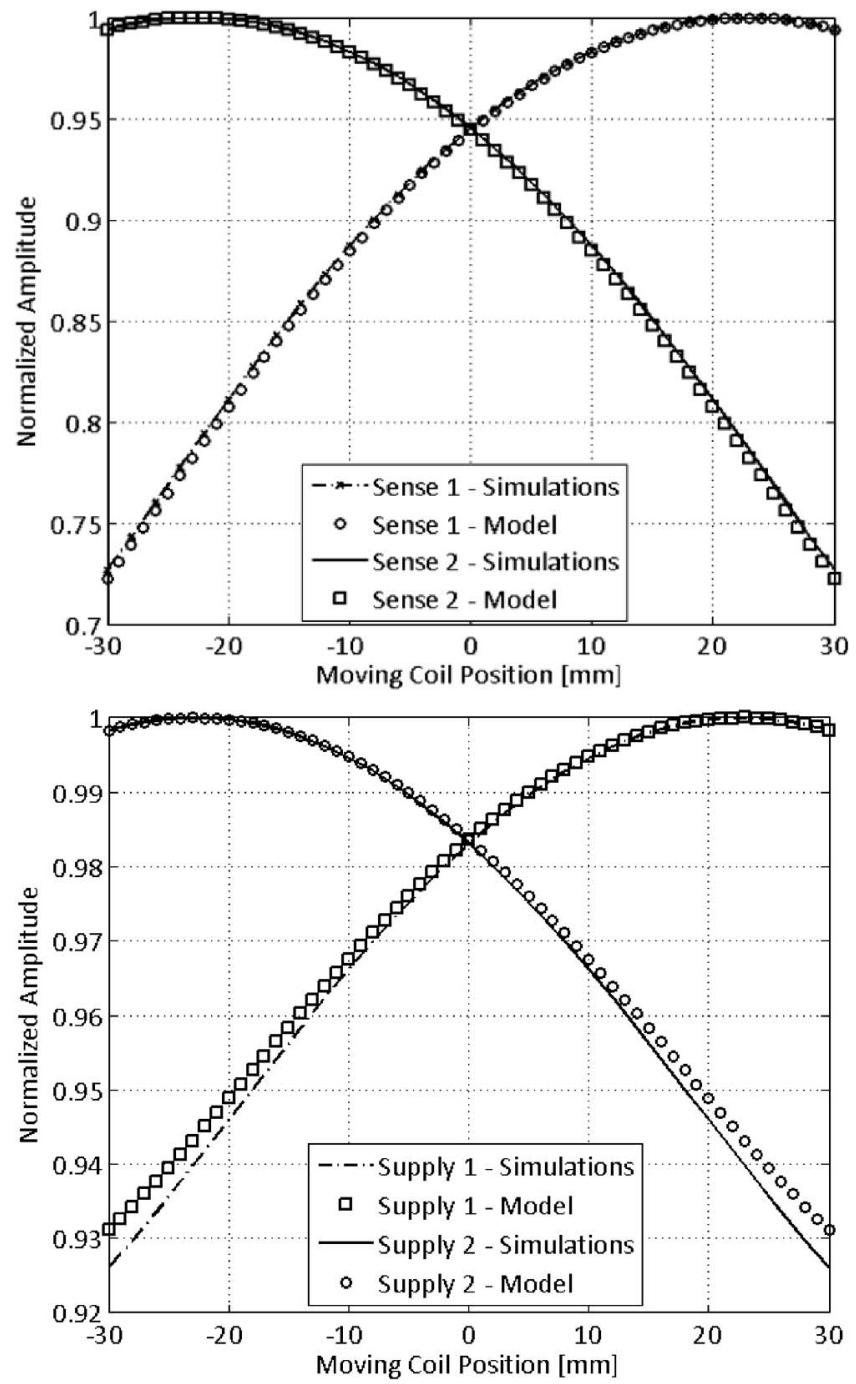

Fig. 5. (Top) Comparison between normalized amplitudes of sense voltages in case of current supply. (Bottom) Comparison between normalized amplitudes of supply voltages for current supply.

support bobbins have been modeled with nonmagnetic and nonconductive materials, which have the same B-H relationship as air. Among all the elements of the simulation structure, the only electrically conducting materials are the five windings, as required for the validation of the model.

The supply of the sensor has been performed through an electrical network simulator, which is coupled with the FEM software and embedded in it. The supply circuits are the same as in Fig. 1.

\section{B. Simulation Results and Discussion}

The excitation is purely sinusoidal, and the fundamental harmonics of the signals have been computed in the analysis. The computational time for the simulations has been around $8 \mathrm{~h}$ on a Windows-based 64-bit 3-GHz quad-core system with 4-GB random access memory. On the other hand, the calculation of the mutual inductances for the model's equations along the entire position range took around $5 \mathrm{~min}$ without approximations, $30 \mathrm{~s}$ adopting a scaling factor of 0.1 . The
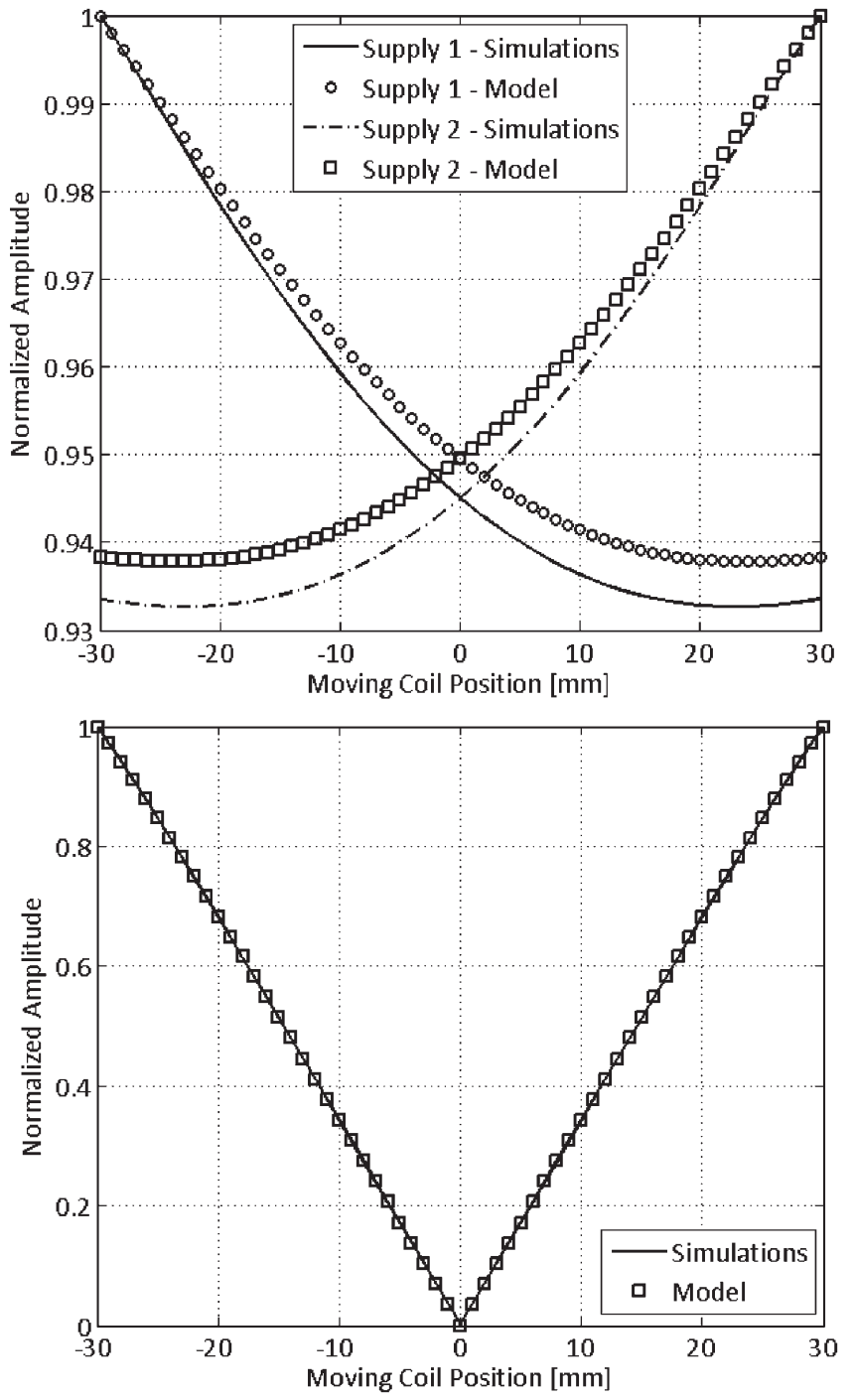

Fig. 6. (Top) Comparison between normalized amplitudes of the supply currents for voltage supply. (Bottom) Comparison between normalized amplitudes of the moving coil induced current for voltage supply.

evaluations of elliptic integrals have been on the order of $10^{9}$ for each position value in the non-approximated computation.

The results are depicted in Fig. 5 for current supply and in Fig. 6 for voltage supply. The magnitudes have been normalized to their maximum value to better highlight the signal dynamics on the position range and to have a direct percentage comparison with respect to the model. However, the percentage agreement with regard to the actual values will be also cited. By doing so, two types of agreement are pointed out, namely, the agreement on the magnitudes (obtained by comparing the values) and the agreement on the trends (obtained by comparing the normalized magnitudes). A comparison, including the numerical values, will be finally proposed in the experimental validation since, in that case, the measurements are done on an actual prototype (i.e., with fixed parameters).

Fig. 5 shows the simulation results with regard to the sense and supply voltages and their comparison with the model in case of current supply. As predicted by the theory, the supply voltages with current supply are also a function of the position. Both graphs describe nonlinear and symmetric dependence of 
the first harmonics with respect to the moving coil position. Such nonlinearity with the position can be minimized through an optimization. If the optimized solution is not suitable (e.g., for space constraints), a calibration with many position points can be done.

The graphs show a very good agreement on the voltage values (more than 97\%) and on the voltage dynamics (always above $95 \%$ ). The voltage swing (i.e., the difference between the sense voltage values at the limits of the position range) can be designed to be of some volts. In these simulations, a value of $1.1 \mathrm{~V}$ has been observed. It is important to notice that the dynamics of the signals is much more relevant on the sense voltages $(27.5 \%$ amplitude change in the considered position range) rather than on the supply voltages (7\% amplitude change in the position range).

The graphs in Fig. 6 illustrate the simulation results with regard to the currents in the supply windings and in the moving coil with voltage supply. It is preferable to look at these magnitudes in voltage supply rather than at the voltages since, with voltage supply, the position dependence is present also in the supply currents. In addition, these currents, together with the induced current in the moving coil, build the sense voltages, as evident from (8). The supply currents behave in a nonlinear way, and the agreement between the simulations and the model is very good in this case, too (more than $98 \%$ for the values and more than $93 \%$ for the dynamics). Once again, the symmetry of the device is evident in the curves. However, the induced current in the moving coil is a linear function of the position. In particular, as the model predicted and the working principle of the sensor required, the current is null when the moving coil is in the center of the structure. The agreement with the model is excellent in this case (more than 98\%).

Both supply cases exhibit a nonlinear behavior of the electrical magnitudes with the moving coil position. This nonlinearity can be studied and justified by looking at the model's equations, i.e., (11) and (15). If the single mutual inductances of the windings are evaluated, through (3), the dependence of the mutual inductance with the position is linear, as moreover explained in [13]. Nevertheless, in (11) and (15), the expressions involve products between mutual inductances. Therefore, the overall relationship between voltages (in current supply) or currents (in voltage supply) with the position, in turn, involve such products, leading to a parabola-like behavior.

For what has been observed, the preliminary validation of the proposed analytical model showed an agreement between the model and the simulations, which is always around $96 \%$ both for values and dynamics. The least agreeing cases are reported for magnitudes, which exhibit the smallest dynamics, such as the supply voltages for current supply and the supply currents for voltage supply.

\section{EXPERIMENTAL VALIDATION}

Experimental measurements on an ironless position sensor prototype have been carried out to further verify the model's results and assess its hypotheses. This point is of crucial importance since the validated model would then be used as a tool for future sensor's design and optimization.

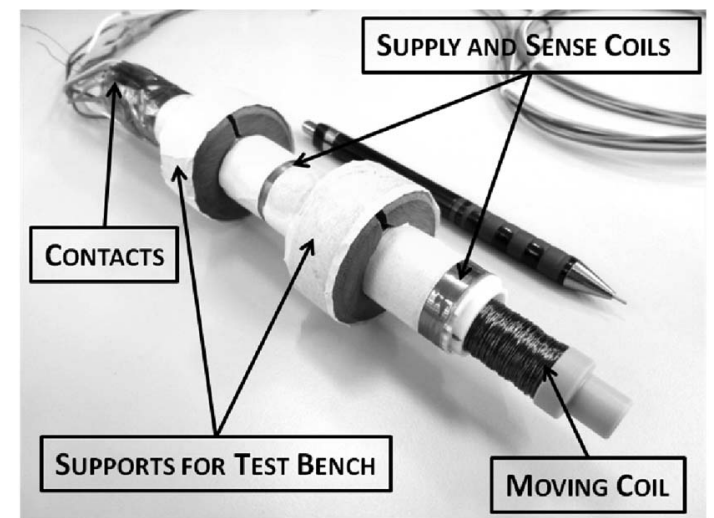

Fig. 7. I2PS prototype with its main parts and supports for the test bench.

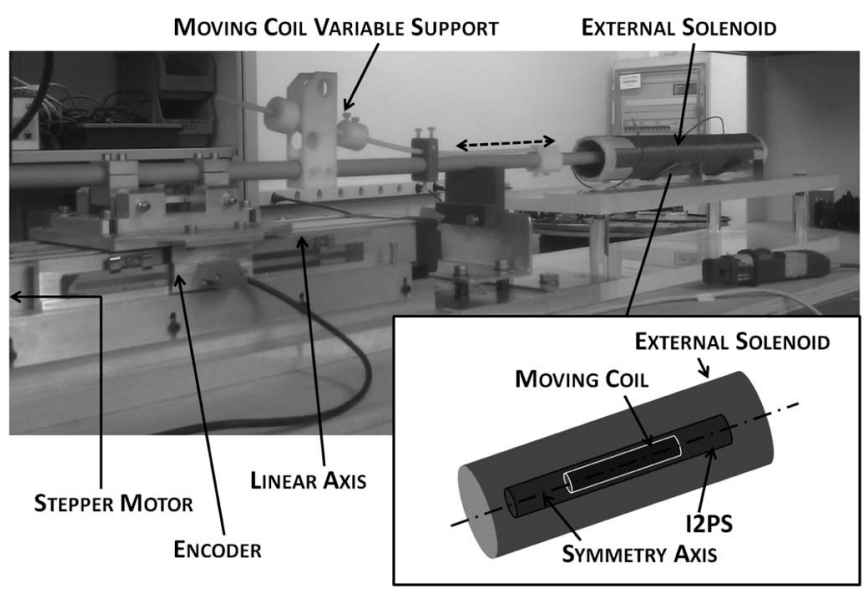

Fig. 8. (Background) Measurement test bench for testing the I2PS. An optical encoder (0.5- $\mu \mathrm{m}$ uncertainty) is used as the reference sensor for the calibration. (Square) Sensor's arrangement in the solenoid, used to hold the sensor and to possibly create an external field.

\section{A. Custom Prototype and Experimental Setup}

The experimental measurements presented here have been carried out on an ironless position sensor prototype, which has been manufactured to validate the model. Once again, the geometrical and electrical parameters (e.g., diameters and number of turns) have not been optimized since the aim of the prototype is to verify the model's theory. The sensor's optimization can be performed at a later stage.

The prototype is shown in Fig. 7. All winding supports are made of plastics (nonconductive and nonmagnetic). The numbers of turns are 3150, 4500, and 1400 for the moving, sense, and supply coils, respectively; whereas the overall sensor's dimensions are 22 and $170 \mathrm{~mm}$ for the diameter and length, respectively. The moving coil has been wound with a thicker wire, in order to limit the resistance and guarantee a reasonable induced current, according to (7). For this reason, as moreover evident in Fig. 7, the moving coil turns out to be the most cumbersome part of the sensor's radial structure.

The prototype has been tested using the test bench depicted in Fig. 8 and fully described in [11]. This test bench has been already used to perform measurements on LVDT and I2PS structures with and without external magnetic fields [7], [12]. 


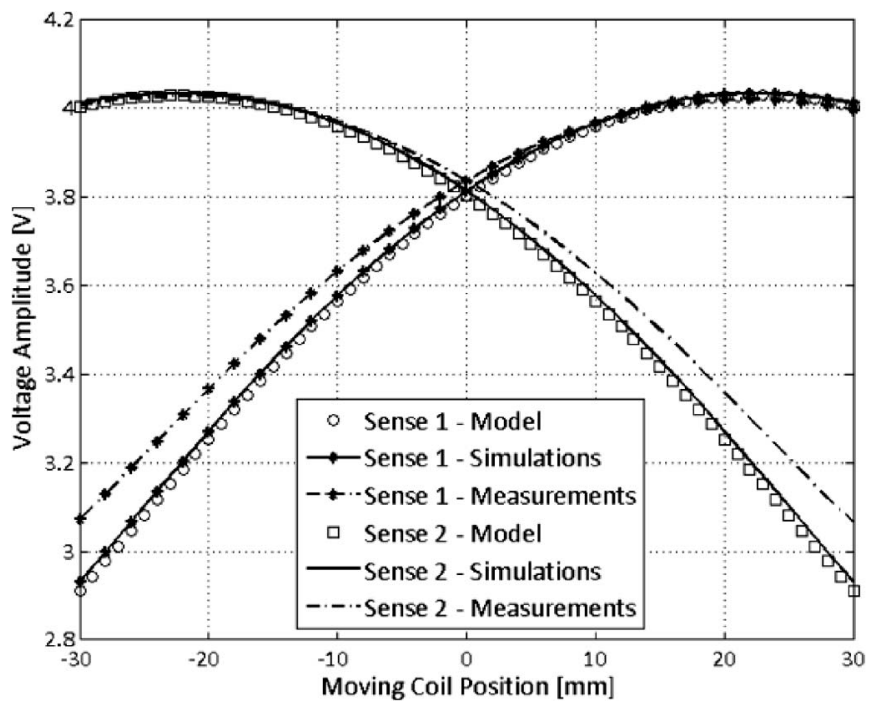

Fig. 9. Comparison of the sense voltages of the ironless position sensor prototype for model, simulations, and measurements with current supply. The maximum measurement uncertainty on the voltages is $70 \mu \mathrm{V}$, obtained as the standard deviation on 30 repeated measurements with a coverage factor of 3 . The current signal is a $25-\mathrm{mA}$ peak sinusoid at $1 \mathrm{kHz}$.

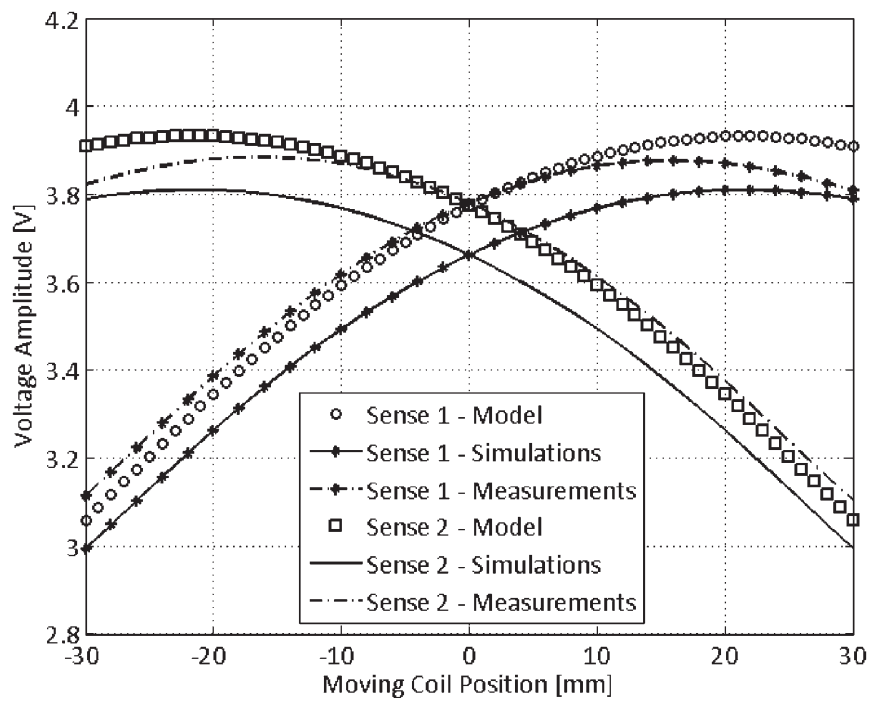

Fig. 10. Comparison of the sense voltages of the ironless position sensor prototype for model, simulations, and measurements with voltage supply. The maximum measurement uncertainty on the voltages is $50 \mu \mathrm{V}$, obtained as the standard deviation on 30 repeated measurements with a coverage factor of 3 . The voltage signal is a $1.85-\mathrm{V}$ peak sinusoid at $1 \mathrm{kHz}$.

\section{B. Experimental Results and Discussion}

The sensor's characteristic curves, showing the amplitude of the sense voltages with respect to the position, are shown in Fig. 9 for current supply and in Fig. 10 for voltage supply. For the sake of work completeness, FEM simulations of the prototype structure have been also performed, and their results have been added as well, in order to have a full overview of the model performances. The simulations have been performed on the same machine as in Section IV.

With current supply, the sensor has been fed with a 25-mA peak sinusoidal signal, whereas with voltage supply, the feeding signal has an amplitude of $1.85 \mathrm{~V}$. The frequency is $1 \mathrm{kHz}$. The choice of these parameters has been done in order to always have the same voltage amplitude on the supply windings when the moving coil is in the null position. This way, a direct comparison between the two cases can be performed.

The model exhibits an excellent agreement with regard to the voltage amplitudes (always above 95\%) and dynamics (above 95\%) for the two supply types, both comparing it with simulations and measurements. With current supply, the sensor exhibits more amplitude dynamics (the swing is about $1.12 \mathrm{~V}$ against $0.85 \mathrm{~V}$ with voltage supply), and this is also successfully predicted by the model. It is also interesting to notice how the nonlinear behavior of the prototype's voltages with the position is perfectly modeled by the analytical equations and evident in current supply, for which the maximum voltage is attained at $22.5 \mathrm{~mm}$. A slight incongruence on the position of the voltage maximum is reported in voltage supply $(20 \mathrm{~mm}$ for simulations and model, $18 \mathrm{~mm}$ for the measurements). This is due to possible incongruences on the primary impedance (given by imperfections of the prototype), which, with voltage supply, yield a slight discrepancy on the supply currents' trend and, in turn, on the sense voltages' dynamics.

\section{CONCLUSION}

The electromagnetic model of an I2PS has been proposed and discussed in this paper. The full mathematical treatise has been shown in both current and voltage supply cases, since both can be used alternatively. An efficient approximation strategy has been also proposed in case of a high computational time for the mutual inductances evaluation. A preliminary validation through FEM simulations has been carried out in order to verify the model's soundness, and the results show a very good agreement. Experimental measurements on a sensor's prototype have been carried out in order to propose a full validation counterpart, and the reported agreement is always above $95 \%$.

This model is then a confirmed powerful tool for the sensor's design process and for quick feedbacks and counterchecks for the sensor's optimization. In this framework, the performance indicators may be the voltage swing, the sensitivity, and the linearity with the position and can be anyway set according to the applications. In addition, the model acts as a theoretical basis for further sensor's characterization, such as the modeling of skin and proximity effects.

\section{ACKNOWLEDGMENT}

The authors would like to thank E. Vire for his support on the measurements; and Dr. M. Markovic, Prof. V. G. Vaccaro, and Prof. F. Cennamo for their fruitful discussions and suggestions.

\section{REFERENCES}

[1] S. Soloman, "Sensors in flexible manufacturing systems," in Sensors Handbook. New York: McGraw-Hill, 2009, pp. 285-288.

[2] I. Sinclair, "Position, direction, distance and motion," in Sensors and Transducers., 3rd ed. Burlington, MA: Newnes, 2003, pp. 33-35.

[3] A. Masi, A. Brielmann, R. Losito, and M. Martino, "LVDT conditioning on the LHC collimators," IEEE Trans. Nucl. Sci., vol. 55, no. 1, pp. 67-75, Feb. 2008.

[4] D. S. Nyce, "The LVDT," in Linear Position Sensors. Hoboken, NJ: Wiley-Interscience, 2004, pp. 94-97. 
[5] Shaevitz LVDTs Provides Rugged Position Sensing for Super Collider, Measurement Specialties Inc., Hampton, VA, 2007. [Online]. Available: http://www.meas-spec.com/assets/0/78/136/497/ a03b1506-410c-4438-83ad-5b9d42bf86c5.pdf

[6] A. Danisi, "Simulation of DC interfering magnetic field effects on the LHC collimators' LVDT positioning sensors," M.S. thesis, Dept. Electron. Eng., Univ. 'Federico II', Naples, Italy, 2009, EDMS Id 1016998.

[7] A. Masi, A. Danisi, R. Losito, M. Martino, and G. Spiezia, "Study of magnetic interference on an LVDT: FEM modeling and experimental measurements," J. Sensors, vol. 2011, pp. 529454-1-529454-9, 2011.

[8] A. Masi and R. Losito, "LHC collimators low level control system," IEEE Trans. Nucl. Sci., vol. 55, no. 1, pp. 333-340, Feb. 2008.

[9] M. Martino, G. Golluccio, R. Losito, and A. Masi, "An analytical model of the effect of external DC magnetic fields on the AC voltages of an LVDT," in Proc. IEEE Int. Instrum. Meas. Technol. Conf., Austin, TX, May 3-6, 2010, pp. 213-218.

[10] M. Martino, A. Danisi, R. Losito, A. Masi, and G. Spiezia, "Design of a LVDT with high rejection to external interfering magnetic field," IEEE Trans. Magn., vol. 46, no. 2, pp. 674-677, Feb. 2010

[11] G. Spiezia, R. Losito, M. Martino, A. Masi, and A. Pierno, "Automatic test bench for measurement of magnetic interference on LVDTs," IEEE Trans. Instrum. Meas., vol. 60, no. 5, pp. 1802-1810, May 2011.

[12] A. Masi, A. Danisi, R. Losito, and Y. Perriard, "Ironless position sensor with intrinsic immunity to external magnetic fields," in Proc. IEEE Sens., Limerick, Ireland, Oct. 28-31, 2011, pp. 2018-2021.

[13] A. Danisi, A. Masi, R. Losito, and Y. Perriard, "Electromagnetic model of an ironless inductive position sensor," in Proc. IEEE I2MTC, Graz, Austria, May 13-16, 2012, pp. 69-74.

[14] K.-B. Kim, E. Levi, Z. Zabar, and L. Birenbaum, "Mutual inductance of noncoaxial circular coils with constant current density," IEEE Trans. Magn., vol. 33, no. 5, pp. 4303-4309, Sep. 1997.

[15] C. Akyel, S. Babic, and S. Kincic, "New and fast procedures for calculating the mutual inductance of coaxial circular coils (circular coil-disk coil)," IEEE Trans. Magn., vol. 38, no. 5, pp. 2367-2369, Sep. 2002.

[16] C. Akyel, S. I. Babic, and M.-M. Mahmoudi, "Mutual inductance calculation for non-coaxial circular air coils with parallel axes," Progr. Electromagn. Res., PIER, vol. 91, pp. 287-301, 2009.

[17] S. Babic and C. Akyel, "Improvement in calculation of the self- and mutual inductance of thin-wall solenoids and disk coils," IEEE Trans. Magn., vol. 36, no. 4, pp. 1970-1975, Jul. 2000.

[18] Flux 9 2D/3D Applications User's Guide, CEDRAT, Meylan, France, 2006.

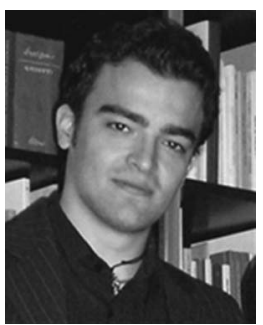

Alessandro Danisi (S'10) was born in Naples, Italy, in 1985. He received the M.Sc. degree in electronic engineering from the University of Naples Federico II, Naples, in 2009. He is currently working toward the Ph.D. degree at the Swiss Federal Institute of Technology Lausanne (EPFL), Lausanne, Switzerland.

In 2007 and 2008, he carried out research activities with regard to Synthetic Aperture Radar simulation in ocean environments and finite-element method (FEM) modeling of electromagnetic sensors. Since 2009, he has been with the Sources, Targets and Interactions Group, Engineering Department, European Organization for Nuclear Research (CERN), Geneva, Switzerland, carrying on research on electromagnetic modeling and characterization of sensors in the presence of magnetic fields. His main research interests are high- and low-frequency electromagnetism and FEM modeling.

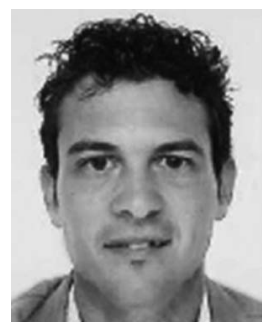

Alessandro Masi was born in Naples, Italy, in 1976. $\mathrm{He}$ received the M.Sc. degree in electronic engineering and the Ph.D. degree in computer science from the University of Naples Federico II, Naples. His $\mathrm{Ph} . \mathrm{D}$. research on high-accuracy measurement systems for superconducting magnets of the new particle accelerator Large Hadron Collider was carried out with the Magnetic Measurements and Tests Group, European Organization for Nuclear Research (CERN), Geneva, Switzerland.

He has been a Researcher with the Inter-University Consortium of Computer Science (CINI), on network architecture for remote measurement and control systems. Then, he was with STMicroelectronics as a Digital Designer for one year. Since April 2005, he has been a Staff Member with the Sources, Targets and Interactions Group, CERN, where, since January 2008, he has been leading the Equipment Controls and Electronics Section, which has important section activities such as design, installation, and maintenance of control systems on different platforms; development of radiation-hard motors and sensors; and precision electronics compatible with long cables and with immunity to electromagnetic interference.

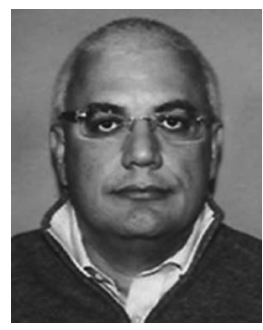

Roberto Losito was born in Naples, Italy. He received the M.Sc. degree in electronic engineering from the University of Naples Federico II, Naples, in 1992.

In 1994, he was a Fellow with the Laboratoire de l'Accélérateur Linéaire, Orsay, France, and with the Institute of Research for Electromagnetics and Electronic Components, National Research Council, Naples. Since 1995, he has been a Staff of the Engineering Department, European Organization for Nuclear Research, Geneva, Switzerland. He participated in the design and construction of radio-frequency systems for the Large Hadron Collider (LHC) for both normal conducting and superconducting systems. Then, he led a group in charge of the design of an LHC collimator low-level control system and a team in charge of compact linear collider photoinjectors. Since 2008, he has been in charge of a group responsible for targets, radioactive ion, and electron sources and studies on the radiation tolerance of control electronics. His research interests include radio frequency, control systems, and particle sources.

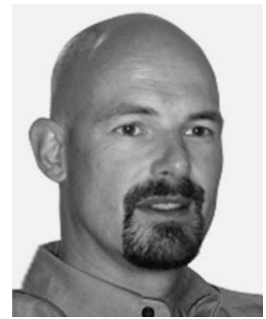

Yves Perriard (SM'05) was born in Lausanne, Switzerland, in 1965. He received the M.Sc. degree in microengineering and the Ph.D. degree from the Swiss Federal Institute of Technology of Lausanne (EPFL), Lausanne, in 1989 and 1992, respectively.

$\mathrm{He}$ was a Co-founder and the Chief Executive Officer of Micro-Beam SA, which is a company involved in high-precision electric drive. In 1998 and since 2003, he was a Senior Lecturer and then has been a Professor, respectively, with EPFL, where he has been the Vice-Director of the Microengineering Institute in Neuchâtel since 2009 and where he is currently the Director of the Laboratory of Integrated Actuators. His research interests are in the field of new actuator design and associated electronic devices.

Prof. Perriard is a member of the Executive Council of the European Power Electronics Association, Brussels; Associate Editor of the IEEE Journal of Electrical Engineering; and Associate Editor of the IEEE TRANSACTIONS ON INDUSTRY APPLICATIONS. 